\title{
Simulation Study of Single Line-to-Ground Faults on Rural Teed Distribution Lines
}

\author{
Wanying Qiu \\ School of Basic Sciences, East China Jiaotong University, Nanchang, P. R. China
}

\begin{abstract}
Teed lines are widely used in rural power networks in China. The paper theoretically analyzes the voltages and currents when a single line-toground fault occurs, constructs a new mathematical model for fault location on the teed lines, performs corresponding MATLAB simulations. Simulation results show that currents near the source (bus $\mathrm{M}$ ) are almost independent of fault positions and fault resistances. However, voltages at bus $\mathrm{M}$ are sensitive to fault positions while fault voltages at fault position are only sensitive to transient resistances. A novel approach to fault location on teed lines is proposed. First, the faulted line is identified with remote control equipment. Then, the transient resistances are calculated according to the fault voltages. And at last, the fault distances are computed according to the voltages at bus $\mathrm{M}$ and the calculated transient resistances.
\end{abstract}

Keywords: simulation, fault location, teed line, single line-to-ground fault.

\section{Introduction}

Power lines are typically teed in rural distribution systems in China. Faults occur when equipment insulation fails, due to system overvoltages, lightning, contamination or other mechanical causes. Single line-to-ground faults account for about $90 \%$ of the total faults according to the statistics[1-4]. Fault location equipment is developed to find and clear faults as soon as possible. Unfortunately, the accuracy and reliability of present location equipment for rural power networks are not satisfactory. Therefore, it is necessary to study the characteristics of faults in rural power networks and lay a solid foundation for developing corresponding fault location equipment.

\section{Theoretical Analysis}

Consider a single line-to-ground fault from phase a to ground at the general three-phase bus shown in Figure 1. For generality, a fault impedance $\mathrm{Zf}$ is included. In the case of a bolted fault, $\mathrm{Zf}=0$, whereas for an arcing fault, $\mathrm{Zf}$ is the arc impedance. In the case of a insulator flashover, Zf equals the total fault impedance between the line and ground, including the impedance of the arc and the post or tower. Since any of the three phases can be arbitrarily labeled phase a, analysis results can be easily applied to phase $b$ or phase c. 
From Figure 1, fault conditions in phase domain are:

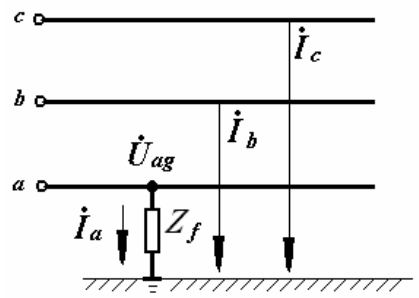

Fig. 1. Single line-to-ground fault

$$
\begin{gathered}
\dot{I}_{b}=\dot{I}_{c}=0 . \\
\dot{U}_{a g}=Z_{f} \dot{I}_{a} .
\end{gathered}
$$

Transforming fault conditions to sequence domain, the sequence components of the fault currents are obtained:

$$
I_{0}=I_{1}=I_{2}=\frac{U_{f}}{Z_{\Sigma}+3 Z_{f}} .
$$

where $U_{f}$ is prefault voltage and $Z_{\Sigma}=Z_{0}+Z_{1}+Z_{2}$.

The fault current on phase a can be derived by transforming the sequence components to the phase domain:

$$
I_{a}=\frac{3 U_{f}}{Z_{\Sigma}+3 Z_{f}} .
$$

The line-to-ground voltages can then be determined from equation (2).

In the case of a bolted fault, $Z_{f}=0$, it can be easily obtained that the line-to-ground voltage $U_{a g}=0$, while $U_{b g}=U_{\mathrm{cg}}=1.732 \mathrm{Uph}$, where $U_{p h}$ is the phase voltage.

\section{MATLAB Simulations}

Typical teed lines are shown in Figure 2.

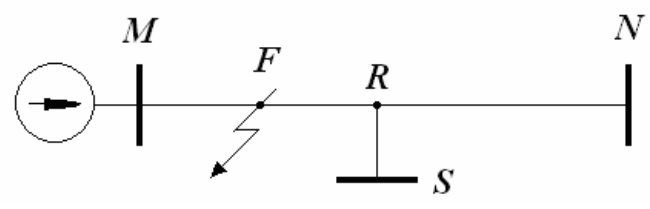

Fig. 2. Typical teed lines

The MATLAB/Simulink simulation circuit is illustrated in Figure 3. 


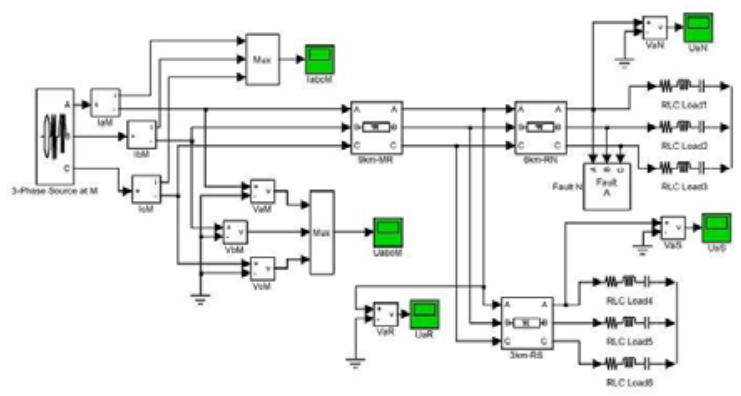

Fig. 3. Simulation circuit

In Figure $2, l_{M R}=9 \mathrm{~km}, l_{R N}=6 \mathrm{~km}, l_{R S}=3 \mathrm{~km}$. Each line is equivalent to a pi-block as shown in Figure 3.

Parameters are from real statistics (written in MATLAB grammar).

Source parameters are:

Phase-to-phase rms voltage: $10500 \mathrm{~V}$; Phase angle of phase A: 0 degree; Frequency: $50 \mathrm{~Hz}$; Internal connection: Y; Source resistance: $0.1638 \mathrm{Ohms}$; Source inductance: $0.000236 \mathrm{H}$.

Loads are: $\mathrm{PAN}=\mathrm{PBN}=\mathrm{PCN}=100000 \mathrm{~W} ; \mathrm{QAN}=\mathrm{QBN}=\mathrm{QCN}=40000 \mathrm{Var}$;

$\mathrm{PAS}=\mathrm{PBS}=\mathrm{PCS}=300000 \mathrm{~W}$; $\mathrm{QAS}=\mathrm{QBS}=\mathrm{QCS}=40000 \mathrm{Var}$.

Line parameters are: $\mathrm{r} 1=0.330 \Omega / \mathrm{km}, \mathrm{L} 1=0.001126 \mathrm{H} / \mathrm{km}, \mathrm{C} 1=9.14 \mathrm{e}-9 \mathrm{~F} / \mathrm{km}$.

Set Rf $=[0,10,20,30,40,50,60,70,80,90,100,200,500,1000] \Omega$, change the fault point one by one, run MATLAB /Simulink/ SimPowerSystems, and many pages of simulation results are acquired. Parts of the results are shown in Table 1.

Table 1. Parts of the Simulation Results

\begin{tabular}{ccccccc}
\hline \multirow{2}{*}{$D / \mathrm{km}$} & \multicolumn{3}{c}{$R_{f}=20 \Omega$} & \multicolumn{3}{c}{$R_{f}=500 \Omega$} \\
& $I_{M} / A$ & $U_{M} / V$ & $U_{F} / V$ & $I_{M} / A$ & $U_{M} / V$ & $U_{F} / V$ \\
\hline 3 & 62.2 & 93.3 & 4.95 & 62.2 & 193 & 125 \\
\hline 6 & 62.2 & 180 & 4.95 & 62.2 & 273 & 124 \\
\hline 9 & 62.2 & 273 & 4.95 & 62.2 & 354 & 123 \\
\hline 15 & 62.2 & 320 & 4.95 & 62.2 & 400 & 122 \\
\hline
\end{tabular}

Table 1 implies that currents through bus $M$ are almost independent of fault positions and fault resistances. In other words, fault currents are of little significance for fault location. However, voltages at bus $M$ are sensitive to fault positions while fault voltages at fault position are only sensitive to transient resistances. Therefore, a new approach to fault location on teed lines is presented as follows. First, the faulted line, $\mathrm{MR}, \mathrm{RN}$, or RS, is identified with remote control equipment. Then, the transient resistances are calculated according to the fault voltages, which are approximately equal 
to voltages at bus $\mathrm{N}$ or bus $\mathrm{S}$. And at last, the fault distances are computed according to the voltages at bus $\mathrm{M}$ and the calculated transient resistances.

Take line MR for example, by using the least square method of MATLAB:

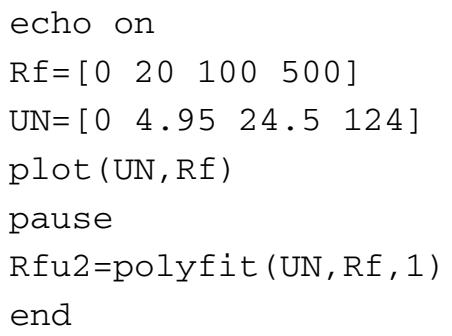

\% fit the Rf-UN curve

The relation between Rf and UF can be obtained as

$$
\mathrm{R}_{\mathrm{f}}=4.0305 \mathrm{U}_{\mathrm{F}}+0.3812 \text {. }
$$

Similarly, the relation among d (fault distance from M), Rf and UM can be also obtained by the least square method.

\section{Conclusions}

Fault currents through bus $\mathrm{M}$ are almost independent of fault distances and fault resistances. While voltages at bus $\mathrm{M}$ are sensitive to fault distances, and fault voltages at fault position are only sensitive to transient resistances. Therefore, a new idea to locate the fault on teed lines is propose. Firstly, identifying the faulted line; secondly, calculating the transient resistances; and at last, computing the fault distances.

\section{References}

[1] Zoran, M.R., Shin, J.-R.: New One Terminal Digital Algorithm for Adaptive Reclosing and Fault Distance Calculation on Transmission Lines. IEEE Trans on Power Delivery 21(3), 1231-1237 (2006)

[2] Venkatesan, R., Balamurugan, B.: A Real-Time Hardware Fault Detector Using Artificial Neural Network for Distance Protection. IEEE Trans. on Power Delivery 16(1), 75-82 (2001)

[3] Sukumar, M.B.: New Fault-Location Method for a Single Multiterminal Transmission Line Using Synchronized Phasor Measurements. IEEE Trans. on Power Delivery 21(3), 1148-1153 (2006)

[4] Salat, R., Osowski, S.: Accurate Fault Location in the Power Transmission Line Using Support Vector Machine Approach. IEEE Trans. on Power Systems 19(2), 979-986 (2004) 